\title{
ATIVIDADE FÍSICA DESEMPENHADA POR TRABALHADORES BRASILEIROS: UMA REVISÃO SISTEMÁTICA
}

\author{
Sabrina Fernandes de Azevedo \\ Universidade Federal de Santa Catarina, Florianópolis, Santa Catarina, Brasil \\ Adair da Silva Lopes \\ Universidade Federal de Santa Catarina, Florianópolis, Santa Catarina, Brasil
}

\begin{abstract}
Resumo
Estudo bibliográfico que objetivou revisar pesquisas na área de atividade física com trabalhadores brasileiros em bases de dados eletrônicas como a Medline, Lilacs e Scielo. Utilizaram-se os seguintes termos em português/inglês: atividade física/physical activity, atividade motora/motor activity, exercício físico/physical exercise combinados com o termo trabalhador/worker, no período entre 2000 e 2010. Nesta primeira etapa, encontrou-se 303 estudos. O processo de seleção dos estudos abrangeu a leitura dos títulos, resumos e textos completos. Após esta fase, 27 estudos cumpriram os critérios de inclusão da revisão. Há uma grande variabilidade metodológica nos estudos nacionais. No entanto, não existe uma homogeneidade no delineamento, sendo necessários estudos longitudinais na área.
\end{abstract}

Palavras-chave: Atividade Motora. Trabalhadores. Brasil.

\section{Introdução}

$\mathrm{O}$ $\mathrm{s}$ benefícios da prática regular de atividade física $(\mathrm{AF})$ para a saúde têm sido cada vez mais destacados em diferentes meios de comunicação. Apesar de serem crescentes as evidências de que um estilo de vida fisicamente ativo traga benefícios para a saúde, a inatividade física em adultos varia entre 10,1\% em Macapá e 20,1\% no Distrito Federal, nas capitais brasileiras (VIGITEL BRASIL 2009). No mesmo estudo, observa-se que em todas as cidades estudadas, os homens praticavam mais atividade física no lazer do que as mulheres.

Em se tratando de trabalhadores, a inatividade física varia entre $53,1 \%$ na região sul e sudeste do Brasil a $59 \%$ na região de São Paulo (CODARIN, et al., 2010; PAPINI, et al., 2010); ressaltando que as atividades predominantemente mecanizadas têm contribuído para a prevalência de hábitos de vida não saudáveis (CODARIN, et al., 2010). 
Cabe salientar que, mais de 50\% dos trabalhadores que desempenham a mesma função laboral por um período de 3 anos, podem vir a sofrer de alguma disfunção musculoesquelética (GERR, et al., 2002) e que uma pessoa fisicamente ativa pode minimizar este quadro e prevenir diversas doenças de cunho cardiovascular, metabólico e hormonal, (SILVA; JUVÊNCIO, 2004).

Estudos representativos e de base populacional com trabalhadores são escassos e os resultados diferem, de acordo com o instrumento utilizado, sexo, função exercida e até mesmo as regiões. Como pode ser visto no estudo realizado com trabalhadores da indústria de Santa Catarina, verifica-se que $46,2 \%$ dos indivíduos não realizam $\mathrm{AF}$ no lazer (BARROS; NAHAS, 2001). Em outros estudos, por exemplo, com servidores de Belo Horizonte, nota-se que $25 \%$ dos homens realizam AF no lazer, enquanto $16,67 \%$ das mulheres realizam $\mathrm{AF}$ e que em ambos os sexos níveis insatisfatórios na bateria de testes de aptidão física relacionada à saúde (SILVA; JUVÊNCIO, 2004) e com professores de São Paulo, 56,6\% realizam AF no lazer.

Sendo assim, até o presente momento, não foi encontrada revisão sistemática, nas bases estudadas, com a finalidade de analisar os estudos de medidas de AF em amostra de trabalhadores brasileiros. Com o intuito de preencher esta lacuna do conhecimento, o objetivo desse estudo foi revisar as pesquisas de prevalência de AF em trabalhadores.

\section{Matérias e Métodos}

Trata-se de um estudo bibliográfico (GIL, 2010), que visa à revisão com base em material já publicado. A revisão sistemática da literatura foi realizada a partir de pesquisa bibliográfica de estudos que verificassem a prevalência de AF em trabalhadores de 2000 a 2010. A busca foi realizada em bases de dados eletrônicas como a Medline, Lilacs e Scielo.

\section{Estratégia de Busca}

Para realização da pesquisa foram utilizados os seguintes termos em português/inglês: atividade física/physical activity, atividade motora/motor activity, exercício físico/physical exercise; combinados com o termo trabalhador/worker. Nesta primeira etapa foram encontrados 303 estudos $($ MEDLINE $=179$, LILACS $=104$ E SCIELO $=20)$. 
Seleção de estudos

Inicialmente, realizou-se a leitura dos títulos a fim de verificar a adequação ao propósito dessa revisão. Quando uma decisão não pôde ser tomada mediante os títulos, recorreu-se ao resumo, e, permanecendo a dúvida, a leitura do artigo, na íntegra, foi realizada. Foram incluídos nessa revisão os estudos: (a) cuja amostra incluía 'trabalhadores'; (b) que utilizassem uma medida de AF; (c) cuja coleta de dados tenha sido realizada no Brasil; (d) estudos originais de pesquisa com seres humanos; (e) publicação entre 2000 a 2010; totalizando em 27 artigos. Optou-se por não incluir estudos de intervenção, teses, dissertações e monografias, visto que a realização de uma busca sistemática das mesmas é inviável logisticamente.

Nos estudos que atenderam os critérios de inclusão, foram analisados os seguintes itens: a) ano de publicação; b) periódico publicado; c) amostra; d) sexo; e) localização estudada; f) função; g) características metodológicas; h) tipo de instrumento; i) classificação; e j) prevalência de AF.

\section{Resultados e Discussão}

Primeiramente, conforme o quadro 1, serão apresentadas as características dos 27 estudos incluídos. Destes, 5 foram publicados em 2008 e nenhum estudo sobre a temática foi encontrado nos anos de 2002 e 2003. Apenas um estudo foi publicado num periódico classificado como Qualis "A", e os demais como "B", na área de Educação Física. Observa-se um aumento na produção de trabalhos sobre AF nos últimos anos. Nahas e Garcia (2010) mencionam que houve um grande crescimento no número de publicações sobre atividade física e saúde no Brasil.

De acordo com o mesmo quadro, verifica-se que o sexo masculino prevaleceu em 11 estudos, além disso, 5 estudos abordaram somente o sexo masculino. Nota-se a prevalência no sexo masculino entre os trabalhadores, porém, em diversos estudos populacionais, observa-se que a inatividade física prevalece o sexo feminino (VIGITEL BRASIL 2009; BAUMAN, et al.,2009; DEL DUCA, et al, 2011).

Quanto à região estudada, vê-se que 12 estudos foram realizados na região sudeste do Brasil, 7 na região sul e apenas dois estudos não Resultado semelhante foi encontrado na revisão de Dumith (2009), 
que teve como objetivo descrever a prevalência de atividade (ou inatividade) física no Brasil. Num levantamento sobre a produção do conhecimento sobre educação física no programa de pós-graduação no Rio Grande do Sul, nota-se que a temática 'atividade física e saúde' foi a mais expressiva nesta região (FRIZZO, 2010) em contrapartida na região nordeste, observa-se que foi a menos expressiva (TAFFAREL; GAMBOA; GAMBOA, 2009). Isto pode ser considerado pelo fato de haver maiores grupos de estudos e programas de pós-graduação nas regiões sudeste e sul (NAHAS; GARCIA, 2010). Salienta-se, portanto, a relevância de incentivo à pesquisa para as demais regiões, principalmente a norte e a centro-oeste, além disso, a necessidade de estudos com amostras representativas na região nordeste.

Diversos tipos de trabalhadores foram considerados por tais pesquisas, entretanto os mais estudados foram os trabalhadores da indústria, e, logo após, os profissionais da saúde.

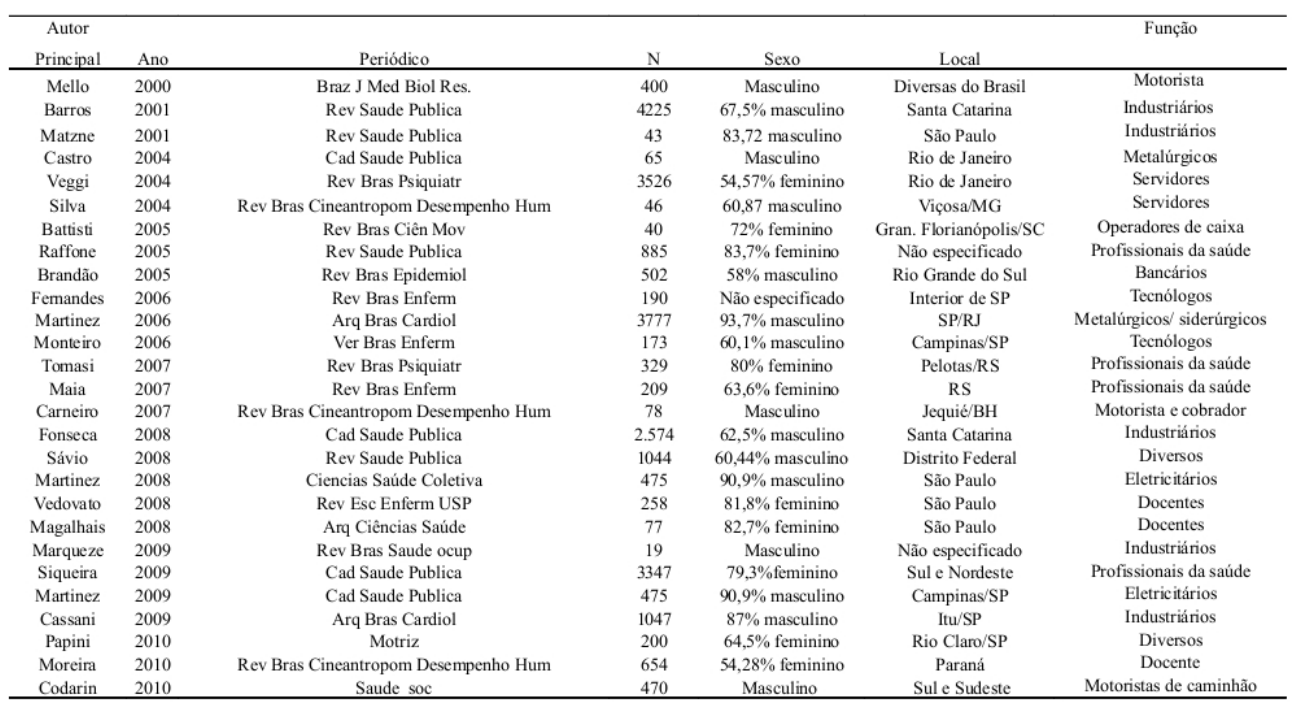

Quadro 1: Características dos artigos incluídos na revisão

As características metodológicas dos estudos, segundo critérios de avaliação previamente estabelecidos, serão apresentadas na tabela 1. Quanto ao tipo de estudo, prevaleceu do tipo descritivo em 20 trabalhos (74,07\%); cuja função é verificar precisamente a incidência e distribuição dos fenômenos de uma determinada área e estabelecer uma relação entre estes fenômenos (GIL, 2010). Em relação à amostra, prevaleceu a não probabilística em 18 estudos (66,67\%). Este tipo de amostra representa por si próprio, e não, uma população; ou seja, não há uma garantia de representatividade (MINATTO, et al., 2011). De 
todos os estudos, apenas $9(33,33 \%)$ mencionam as informações sobre a testagem prévia dos instrumentos e $11(40,74 \%)$ o fazem com relação à validade destes. Observa-se que $17(62,96 \%)$ falam sobre as variáveis do estudo e $27(100 \%)$ sobre a atividade física. Nota-se ainda que, 22 estudos $(81,48 \%)$ citam os aspectos éticos, fato este, que pode ser considerado pela exigência por parte dos periódicos.

Tabela 1: Características dos artigos segundo aspectos metodológicos

\begin{tabular}{lcc}
\hline CARACTERISTICA METODOLÓGICA & $\mathrm{N}$ & $\%$ \\
\hline TIPO DE ESTUDO & 20 & 74,07 \\
Descritivo & 5 & 18,52 \\
Epidemiológico & 2 & 7,41 \\
Não reportam & 9 & 33,33 \\
AMOSTRA & 18 \\
Probabilística & 66,67 \\
Não-Probabilística & 9 \\
INSTRUMENTO & 33,33 \\
Informações sobre testagem prévia do instrumento & 41 \\
Informações sobre validade do instrumento & 40,74 \\
MEDIDAS & 17 \\
Definição operacional das variáveis & 62,96 \\
Mensuração da atividade física & 27 \\
ASPECTOS ÉTICOS & 100 \\
Informações sobre aspectos éticos* & 22 \\
\hline
\end{tabular}

*Menção de aprovação em comitê de ética, anonimato e termo de consentimento livre e esclarecido.

Por fim, pondera-se na tabela 2, os métodos de mensuração, bem como a sua classificação e os resultados encontrados. Sendo que, apenas um estudo utilizou dois métodos, envolvendo um questionário e a bateria de teste de Aptidão Física Relacionada à Saúde (AFRS). O restante utilizou apenas um método, sendo que 23 estudos um questionário, dois estudos a taxa metabólica basal e um estudo uma entrevista. Quanto ao instrumento, verifica-se uma predominância em questionários. A vantagem deste método está no seu baixo custo financeiro, baixa demanda de tempo de pesquisa e na possibilidade de seu uso em estudos amplos (REIS; PETROSKI; LOPES, 2000). Todavia, não há uma padronização metodológica no delineamento, instrumento e definição das variáveis, fato que dificulta a comparação dos estudos, inclusive no que tange aos resultados. Situação similar foi encontrada em outras revisões, dentre as quais, uma com o objetivo de analisar as medidas de AF e comportamentos sedentários em adolescentes brasileiros (TASSITANO, et al., 2007), e outra, com o intuito de verificar a prevalência de sobrepeso/obesidade em adolescentes brasileiros (ARAUJO, et al., 2007). 
Quanto ao resultado encontrado, há uma variabilidade de resultados devido à região estudada, sexo, instrumento e até mesmo ponto de corte.

A atividade física é um dos fatores mais importantes para melhorar a saúde da população, contudo, não existe um sistema padronizado para vigilância internacional (BAUMAN, et al., 2009). Sendo assim, necessita-se aprimorar os instrumentos de medida, e, melhorar a sua precisão e condições de utilização em estudos amplos (REIS; PETROSKI; LOPES, 2000). Em nosso país, a qualidade da produção científica, na área de atividade física e saúde, ainda precisa ser aperfeiçoada - assim como em todas as demais áreas relacionadas à Educação Física (NAHAS; GARCIA, 2010).

Tabela 2: Características dos instrumentos, definição operacional das variáveis e resultados encontrados.

\begin{tabular}{|c|c|c|c|}
\hline Primeiro Autor & Instrumento & Classificação & Resultado \\
\hline Mello & Questionánio & Não apresenta & $58,8 \%$ não realizam $\mathrm{AF}$ \\
\hline Barros & Questionário & Inativos, pouco ativos e ativos & $46,2 \%$ não realizam $A F$ no lazer \\
\hline Matzne & Questionário & Não apresenta & 32,56 realizam EF \\
\hline Castro & Questionário & Não apresenta & $52 \%$ realizam AF lazer \\
\hline Veggi & Questionário & Não apresenta & $26,3 \% \mathrm{M} \mathrm{e} 25,8 \% \mathrm{~F}$ não realizam $\mathrm{AF}$ no lazer \\
\hline Silva & Quest. e Bateria de teste AFRS* & Não apresenta & $25 \% \mathrm{M} \mathrm{e} 16,67 \% \mathrm{~F}$ realizam $\mathrm{AF} /$ ambos os sexos $\mathrm{NI} / \mathrm{p} / \mathrm{a}$ saúde na flexibilidade de tronco \\
\hline Battisti & Questionário & Não apresenta & $57 \%$ realizam AF lazer \\
\hline Raffone & Questionário & Não apresenta & 62,4 não realizam $\mathrm{AF}$ lazer \\
\hline Brandão & Questionário & Não apresenta & $68 \%$ realizam AF lazer \\
\hline Fernandes & Questionário & Não apresenta & $62,6 \%$ realizam AF lazer \\
\hline Martinez & Questionário & Nenhum tipo de AF e algum tipo de AF & $63,6 \%$ não praticam nenhum tipo de $\mathrm{AF}$ \\
\hline Monteiro & Questionário & Não apresenta & $69,4 \%$ realizam AF lazer \\
\hline Tomasi & Questionário & Não apresenta & $61 \%$ não realizam $\mathrm{AF}$ regular no último mês \\
\hline Maia & Questionário & AF intensa, moderada, leve e inativo & $56 \%$ inativos \\
\hline Carneiro & Questionário & Insuficientemente ativo $\mathrm{e}$ ativo & $60 \%$ insuficientemente ativos \\
\hline Fonseca & Questionário & Não apresenta & $69,2 \%$ ativos no lazer \\
\hline Sávio & Dispêndio energético & Não apresenta & $56 \%$ inativos \\
\hline Martinez & Questionánio & Não apresenta & $71,6 \%$ praticam AF \\
\hline Vedovato & Questionário & Não apresenta & $56,6 \%$ realizam $\mathrm{AF}$ \\
\hline Magalhais & Questionário & Não apresenta & $62,7 \%$ raramente praticam $\mathrm{AF}$ \\
\hline Marqueze & Dispêndio energético & Não apresenta & Valores $<$ ao recomendado de $2000 \mathrm{kcal} / \mathrm{semana} \mathrm{AF}$ no lazer \\
\hline Siqueira & Questionário & Sedentários $<150 \mathrm{~min} / \mathrm{semana}$ & $27,5 \%$ sedentários \\
\hline Martinez & Questionário & Não apresenta & $71,6 \%$ praticam $\mathrm{AF}$ regularmente \\
\hline Cassani & Entrevista & Sedentarismo $<30 \mathrm{~min} / 3 \mathrm{x}$ semana & $83 \%$ sedentários \\
\hline Papini & Questionário & Ativos $\mathrm{e}$ inativos & $59 \%$ inativos no lazer \\
\hline Moreira & Questionário & Não faz parte do seu EV, às vezes e sempre & $52,7 \%$ consideram que AF sempre faz parte do seu EV \\
\hline Codarin & Questionário & Muito ativo, ativo e insuficientemente ativo & $53,1 \%$ insuficientemente ativo \\
\hline
\end{tabular}

\section{Considerações finais}

Conclui-se que há uma crescente na produção científica de estudos relacionados à $\mathrm{AF}$ e ao trabalhador, e, também, uma predominância na região sudeste e sul do Brasil. Quanto ao instrumento de medida para avaliar a $\mathrm{AF}$, prevaleceu à utilização do questionário, mas não há uma padronização metodológica no delineamento, instrumento e definição das variáveis, o que dificulta a comparação dos estudos, inclusive dos resultados. Ressalta-se a necessidade de estudos de prevalência de AF em trabalhadores com amostras representativas. 


\title{
Physical activity performed by Brazilian workers: a systematic review
}

\begin{abstract}
This bibliographic study aimed to review researches on physical activity performed by Brazilian workers, through electronic databases such as Medline, Lilacs, and Scielo. The following terms were used in Portuguese/English: atividade física/physical activity, atividade motora/motor activity, exercício físico/physical exercise combined with the term trabalhador/worker; in the period of 2000 and 2010. First, 303 studies were found. The selection process involved the reading of titles, abstracts, and texts. After that, 27 studies matched the review inclusion criteria. There is a great methodological variability in national studies. Nevertheless, there is no homogeneity in delineament, demanding longitudinal studies in this area.
\end{abstract}

Keywords: Motor Activity. Workers. Brazil.

\section{Actividad física por los trabajadores de Brasil: una revesión sistemática}

\section{Resumen}

Estudio bibliográfico que tuve el objetivo de revisar estudios en el área de actividad física con trabajadores brasileños, en bases de datos como la Medline, Lilacs y Scielo. Los siguientes termos fueran utilizados en portugués/inglés: atividade físi$\mathrm{ca} /$ physical activity, atividade motora/motor activity, exercício físico/physical exercise combinados con el termo trabalhador/worker; entre los años 2000 y 2010 . En esta primera etapa se encontraran 303 estudios. El proceso de selección de los estudios engloba la lectura de los títulos, resúmenes y el texto completo. Después de esta etapa, 27 estudios cumplieron los criterios de inclusión de la revisión. Tienen una grande variabilidad metodológica en los estudios nacionales. Pero, no existe una homogeneidad en el delineamiento, siendo necesarios estudios longitudinales en el área.

Palabras clave: Actividad Motora. Trabajadores. Brasil.

\section{Referências}

ARAÚJO, V. C. et al. Prevalência de excesso de peso em adolescentes brasileiros: um estudo de revisão sistemática. Revista Brasileira de Atividade Física \& Saúde, v. 12, n. 3, p. 79-87, 2007.

BARROS, M. V. G.; NAHAS, M. V. Comportamentos de risco, autoavaliação do nível de saúde e percepção de estresse entre trabalhadores da indústria. Revista de Saúde Pública, vol. 35, n. 6, p. 554-563, 2001.

BAUMAN, A. et al. The International Prevalence Study on Physical Activity: results from 20 countries. International Journal of Behavioral Nutrition and Physical Activity, v. 6, n. 21, p. 1-12, 2009. 
BATTISTI, H. H.; GUIMARÃES, A. C. A.; SIMAS, J. P. N. Atividade Física e Qualidade de Vida de Operadores de Caixa de Supermercado. Revista Brasileira de Ciência e Movimento, v. 13, n. 1, p. 71-78, 2005.

BRANDAO, A. G.; HORTA, B. L.;TOMASI, E. Sintomas de distúrbios osteomusculares em bancários de Pelotas e região: prevalência e fatores associados. Revista Brasileira de Epidemiologia. v. 8, n. 3, p. 295-305, 2005.

BRASIL. Ministério da Saúde. Vigitel Brasil 2009: vigilância de fatores de risco e proteção para doenças crônicas por inquérito telefônico. Secretaria de Vigilância em Saúde, Secretaria de Gestão Estratégica e Participativa. Brasília, 2010.

CARNEIRO, L. R. V.; COQUEIRO, R. S.; FREIRE, M. O.; BARBOSA, A. R.. Sintomas de distúrbios osteomusculares em motoristas e cobradores de ônibus. Revista Brasileira de Cineantropometria \& Desempenho Humano, v. 9, n. 3, p. 277-283, 2007.

CASSANI, R. S. L.; NOBRE, F.; PAZIN FILHO, A.; SCHMIDT, A. Prevalência de fatores de risco cardiovascular em trabalhadores de uma indústria brasileira. Arquivos Brasileiros de Cardiologia, v. 92, n. 1, p. 16-22, 2009.

CASTro, M. B. T.; ANJOS, L. A.; LOURENCO, P. M. Padrão dietético e estado nutricional de operários de uma empresa metalúrgica do Rio de Janeiro, Brasil. Cadernos de Saúde Pública, v. 20, n. 4, p. 926-934, 2004.

CODARIN, M. A. F. et al. Associação entre prática de atividade física, escolaridade e perfil alimentar de motoristas de caminhão. Saúde e Sociedade, v. 19, n. 2, p. 418-428, 2010.

DEL DUCA, G. F.; OLIVEIRA; E. S. A.; SOUSA; T. F.; SILVA; K. S.; NAHAS, M. V. Inatividade física no lazer em trabalhadores da indústria do Rio Grande do Sul, Brasil. Motriz, Rio Claro, v. 17 n. 1, p. 180-188, jan./mar. 2011.

DUMITH, S. C. Physical activity in Brazil: a systematic review. Cadernos de Saúde Pública, vol. 25, suppl. 3, p. S415-S426, 2009. 
FERNANDES, A. C. P.; MONTEIRO, M. I. Capacidade para o trabalho entre trabalhadores de um condomínio de empresas de alta tecnologia. Revista Brasileira de Enfermagem, v. 59, n. 6, p. 752-756, 2006.

FRIZZO, G. F. E. A produção do conhecimento da Educação Física no programa de Pós-Graduação em Ciências do Movimento Humano da UFRGS. Pensar a Prática, Goiânia, v. 13, n. 3, p. 116, set./dez, 2010.

FONSECA, S.A.; BLANK, V. L. G.; BARROS, M. V.; NAHAS, M. V. Percepção de saúde e fatores associados em industriários de Santa Catarina, Brasil. Cadernos de Saúde Pública, v. 24, n. 3, p. 567-576, 2008.

GERR F. et al. A prospective study of computer users: I. Study design and incidence of musculoskeletal symptoms and disorders. American Journal of Industrial Medicine, v. 41, p. 221-235, 2002.

GIL, A C. Como elaborar projetos de pesquisa. 5 ed. São Paulo: Atlas, 2010.

MAGALHAIS, L. C. B.; YASSAKA, M. C. B.; SOLER, Z. A. S. G. Indicadores da qualidade de vida no trabalho entre docentes decurso de graduação em enfermagem. Revista Arquivos de Ciência da Saúde, v. 15, n. 3, p. 117-124, 2008.

MAIA, C. O. et al. Fatores de risco modificáveis para doença arterial coronariana nos trabalhadores de enfermagem. Acta Paulista de Enfermagem, v. 20, n. 2, p. 138-142, 2007.

MARQUEZE, E. C.; SILVA, M. J.; MORENO, C. R. C. Qualidade de sono, atividade física durante o tempo de lazer e esforço físico no trabalho entre trabalhadores noturnos de uma indústria cerâmica. Revista Brasileira Saúde Ocupacional, v. 34, n. 119, p. 93-100, 2009.

MARTINEZ, M. C.; LATORRE, M. R. D. O. Fatores de risco para hipertensão arterial e diabete melito em trabalhadores de empresa metalúrgica e siderúrgica. Arquivos Brasileiros de Cardiologia, v. 87, n. 4, p. 471-479, 2006. 
. Fatores associados à capacidade para o trabalho de trabalhadores do Setor Elétrico. Cadernos de Saúde Pública, v. 25, n. 4, p. 761-772, 2009.

. Saúde e capacidade para o trabalho de eletricitários do Estado de São Paulo. Revista Ciência \& Saúde Coletiva, v. 13, n. 3, p. 1061-1073, 2008.

METZNER, R. J.; FISCHER, F. M. Fadiga e capacidade para o trabalho em turnos fixos de doze horas. Revista de Saúde Pública, v. 35, n. 6, p. $548-553,2001$.

MELLO, M. T. et al. Sleep patterns and sleep-related complaints of Brazilian interstate bus drivers. Brazilian Journal of Medical and Biological Research, v. 33, n. 1, p. 71-77, 2000.

MINATTO, G.; et al. População e amostra / sujeitos da pesquisa. In: SANTOS, S.G. (org.) Métodos e técnicas de pesquisa quantitativa aplicada à educação física. Florianópolis: Tribo da Ilha, 2011. p. 240.

MONTEIRO, M. I.; FERNANDES, A .C. P. Capacidade para o trabalho de trabalhadores de empresa de tecnologia da informação. Revista Brasileira de Enfermagem, v.59, n.5, p. 603-608, 2006.

MOREIRA, H. R.; NASCIMENTO, J. V.; SONOO, C. N.; BOTH, J. Qualidade de vida do trabalhador docente em Educação Física do estado do Paraná, Brasil. Revista Brasileira de Cineantropometria \& Desempenho Humano, v. 12, n. 6, p.435-442, 2010.

NAHAS, M. V.; GARCIA, L. M. T. Um pouco de história, desenvolvimentos recentes e perspectivas para a pesquisa em atividade física $\mathrm{e}$ saúde no Brasil. Revista Brasileira de Educação Física e Esporte, v. 24, n. 1, p. 135-148, 2010.

PAPINI, C. B.; NAKAMURA, P. M.; MARTINS, C. O.; KOKUBUN, E. Severidades Ocupacionais associadas à inatividade física no lazer em trabalhadores. Motriz, Rio Claro, v. 16 n. 3 p.701-707, jul./set. 2010 .

RAFFONE, A. M.; HENNINGTON, E. A. Avaliação da capacidade funcional dos trabalhadores de enfermagem. Revista de Saúde Pública, v. 39, n. 4, p. 669-676, 2005. 
REIS, R. S.; PETROSKI, E. P.; LOPES, A. S. Medidas da atividade física: revisão de métodos. . Revista Brasileira de Cineantropometria \& Desempenho Humano, v. 2, n. 1, p.89-96, 2000.

SAVIO, K. E. O.; COSTA, T. H. M.; SCHMITZ, B. A. S.; SILVA, E. F. Sexo, renda e escolaridade associados ao nível de atividade física de trabalhadores. Revista de Saúde Pública, v. 42, n. 3, p. 457-463, 2008 .

SILVA, C. D.; JUVÊNCIO, J. F. Diagnóstico da aptidão física relacionada à saúde em trabalhadores de escritório da universidade federal de Viçosa. Revista Brasileira de Cineantropometria \& Desempenho Humano, v. 6, n. 1, p. 63-71, 2004.

SIQUEIRA, F. C. V. et al. Atividade física em profissionais de saúde do Sul e Nordeste do Brasil. Cadernos de Saúde Pública, v. 25, n. 9 , p. 1917-1928, 2009.

TASSITANO, R. M. et al. Atividade física em adolescentes brasileiros: uma revisão sistemática. Revista Brasileira de Cineantropometria \& Desempenho Humano, v. 9, n. 1, p.55-60, 2007.

TOMASI, E. et al. Condições de trabalho e automedicação em profissionais da rede básica de saúde da zona urbana de Pelotas, RS. Revista Brasileira de Epidemiologia, v. 10, n. 1, p. 66-74, 2007.

VEDOVATO, T. G.; MONTEIRO, M. I. Perfil sociodemográfico e condições de saúde e trabalho dos professores de nove escolas estaduais paulistas. Revista da Escola de Enfermagem da USP, v. 42, n. 2, p. 291-297, 2008.

VEGGI, A. B.; LOPES, C. S.; FAERSTEIN, E.; SICHIERI, R. Índice de massa corporal, percepção do peso corporal e transtornos mentais comuns entre funcionários de uma universidade no Rio de Janeiro. Revista Brasileira de Psiquiatria, v. 26, n. 4, p. 242-247, 2004.

TAFFAREL, C. N. Z; GAMBOA, M. C.; GAMBOA, S. A. S. A produção do conhecimento em Educação Física nos Estados de Alagoas, Bahia, Pernambuco e Sergipe (1982-2004). Filosofia e Educação, v. 1, n. 1, p.164-167, 2009. 
Recebido em: 15/04/2011

Revisado em: 24/09/2011

Aprovado em: 07/02/2012

Endereço para correspondência

adair@cds.ufsc.br

Adair da Silva Lopes Correio

Universidade Federal de Santa Catarina

Centro de Desportos, Departamento de Educação Física.

Campus Universitário - Trindade

Trindade

88040-900 - Florianopolis, SC - Brasil 